\title{
Aspects of Gravity's Rainbow in Black Hole Entropy ${ }^{\dagger}$
}

\section{Remo Garattini}

University of Bergamo, Bergamo, Italy

+ Presented at the Entropy 2021: The Scientific Tool of the 21st Century, 5-7 May 2021; Available online: https://sciforum.net/conference/Entropy2021/.

Published: 5 May 2021

Quantum Field Theory is plagued by divergences in the attempt to calculate physical quantities. Standard techniques of regularization and renormalization are used to keep under control such a problem. Gravity's Rainbow seems to offer a different scheme which is able to remove infinities when Black Hole Entropy is computed in contrast to what happens in conventional approaches. In particular, we apply the Gravity's Rainbow regularization scheme to the computation of the entropy of a Schwarzschild black hole from one side. In a second step, we will consider the effects of rotations on the calculation of some thermodynamical quantities like the free energy, internal energy and entropy. Even in this case, in ordinary gravity, when we evaluate the density of states of a scalar field close to a black hole horizon, we obtain a divergent result which can be kept under control with the help of some standard regularization and renormalization processes. Once again we will show that when we use the Gravity's Rainbow approach such regularization/renormalization processes can be avoided. A comparison between the calculation done in an inertial frame and in a comoving frame is presented.

(C) 2021 by the author. Licensee MDPI, Basel, Switzerland. This article is an open access article distributed under the terms and conditions of the Creative Commons Attribution (CC BY) license (http://creativecommons.org/licenses/by/4.0/). 